\title{
Comparison of the Effects of General and Pregnant Women's Dentifrices on the Removal of the Dental Plaque
}

\author{
Ye-Eun Kim, Seong-Eun Ko, Da-Eun Sa, Ji-Eun Lee, Se-Yoon Jeon, and Do-Seon Lim ${ }^{\dagger}$ \\ Department of Dental Hygiene, College of Health Science, Eulji University, Seongnam 13135, Korea
}

\begin{abstract}
Background: This study tries to compare and analyze the removal effect of dental plaque of general dentifrice and pregnant women's dentifrice and quantify the results to provide basic data so that consumers can make reasonable choices when purchasing dentifrice, and also increase interest in the dental plaque.

Methods: After forming a dental plaque (carbohydrate porridge) on the labial surface of the bovine teeth, a disclosing agent was applied. Then the same experimenter brushed the surface of the bovine teeth using an electric toothbrush and took photographs using a DSLR camera. Thereafter, the residual amount of dental plaque was analyzed using the Image J program, and SPSS 26.0 was used for statistical processing.

Results: The average residual amount of dental plaque using the general dentifrice was $11.71 \%$ for Perio, $9.45 \%$ for Cliden, and $8.47 \%$ for 2080 , and the average residual amount for the three types was approximately $9.88 \%$. The average residual amount of dental plaque of pregnant women's dentifrice was $13.95 \%$ for Jeninmothers, $12.53 \%$ for Tntnmoms, and $12.63 \%$ for Mommiracle, and the average residual amount of the three types was approximately $13.04 \%$. On comparing the average residual amount of dental plaque between general and pregnant women's dentifrices, it was observed to be $3.16 \%$ higher for the latter. However, the results were not statistically significant.

Conclusion: According to the research results, there was no significant difference in removal effects of general dentifrice and pregnant women's dentifrice. In addition, when a pregnant woman uses the right toothbrushing method with pregnant women's dentifrice, it can prevent or inhibit the progression of the gestational periodontal disease. Therefore, we recommend pregnant women to use pregnant women's dentifrices.
\end{abstract}

Key Words: Bovine teeth, Carbohydrate porridge, Dental plaque, Dentifrice, Pregnant women

\section{Introduction}

Pregnant women become physically and mentally vulnerable and sensitive owing to the changes in endocrine systems. Pregnancy induces changes in the hormonal balance and with the growth of the fetus, the heart exudation, blood volume, and oxygen consumption increase while liver function, lung capacity, and kidney plasma levels decrease in the mother's body. This results in a decrease in the glomerular filtration rate, which increases sensitivity to tension and stress, and decreases resistance to infection ${ }^{1)}$. In particular, the increase in progesterone hormone has an affinity with the underlying substances of connective tissue that make up the gingiva, and increases the osmotic pressure of capillaries distributed in the gingiva, thereby creating a conditions that can easily cause inflammation of the gingiva. Periodontal tissue acts as a reservoir for bacteria and inflammatory immune intermediates, affecting them through blood vessels, and as a result, the risk of developing periodontitis in pregnant women increased by more than twice ${ }^{2)}$. Pregnant women are not only emotionally sensitive but also physically tired, thus neglecting their oral hygiene care. Studies have shown that changes in hormone and oral hygiene control 
behaviors in pregnant women may alter the level of Streptococcus mutans, the main strain causing dental caries, and that these changes may affect the oral cavity of the fetus after childbirth ${ }^{3)}$. Therefore, creating a healthy oral environment and maintaining proper oral hygiene is the most important factor for pregnant women.

Meanwhile, pregnant women's dentifrices targeting pregnant women as the main consumers are available in the market. Dentifrices are auxiliary detergents used to clean the teeth surface efficiently while brushing. Dentifrice, also known as toothpaste, belongs to the sanitary aid, a classification such as soap and detergent, and not medicine. The main ingredients of these detergents are polishing agent, detergents, binders, and wetting agents, and other ingredients include water, flavouring agents, sweeteners, preservatives, prophylactic agents, and treatments. Fluoride is also added as a component that is mixed with dental caries, and fluoride is combined with enamel to increase the acid resistance of the hemorrhoids while brushing teeth, thus preventing dental caries. Sodium lauryl sulfate (SLS) as a synthetic detergent, which is currently most commonly mixed with dentifrices, has some antibacterial properties and reduces surface tension, allowing dentifrices to flow to the surface of the teeth, and is most compatible with the components of other dentifrices, making them more economical, and thus the chemical that is most compatible with the dentifrices ${ }^{4)}$. Studies have demonstrated that using dentifrices containing SLS causes inflammation of the oral mucous membrane, and that it causes inflammatory reactions such as in- flammation of the $\mathrm{skin}^{5)}$, taste change ${ }^{6)}$, and recurrent aphthous stomatitis ${ }^{7)}$. The United States Toxicology study observed that SLS is easily absorbed through the skin and remains in major organs such as the heart, liver and lungs for a certain period, and accumulate in the body for a long duration $^{8)}$. Additionally, previous studies have reported that exposure to fluoride in fetuses would not only impede cognitive development in children but also increase the incidence of attention deficit hyperactivity dissorder ${ }^{9,10)}$. According to these findings, the pregnant women's dentifrices currently available in the market do not contain SLS and fluoride, common in general dentifrices, and are being marketed by replacing the chemical compounds of general dentifrices with natural organic compounds, which require special self-regulatory health care.

Therefore, this study compared the dental plaque removal effect of pregnant women's dentifrice and general dentifrice and analyzed the results to provide basic data to allow pregnant women to make rational choices while purchasing dentifrice.

\section{Materials and Methods}

\section{Research materials}

This study recently selected and used 60 permanent incisor with normal enamel surface among extracted bovine teeth. Six types of dentifrices were selected and divided into control and experimental groups. Three types of general dentifrices were selected as the control group (2080 original- $\alpha$ blue [Aekyung, Seoul, Korea], Perio

Table 1. Appliance of Toothpaste of This Study

\begin{tabular}{|c|c|c|c|c|}
\hline Classification & Group name & Toothpaste name & Active ingredient & $\begin{array}{l}\text { Country of } \\
\text { manufacture }\end{array}$ \\
\hline \multirow[t]{3}{*}{ General toothpaste } & Control group & 2080 original- $\alpha$ blue & Dental type silica, Tocopheryl acetate, Sodium fluoride & Korea \\
\hline & & Perio cavity care & $\begin{array}{l}\text { Tocopheryl acetate, Sodium monofluorophosphate, } \\
\text { Calcium carbonate }\end{array}$ & Korea \\
\hline & & Cliden original & $\begin{array}{l}\text { Dental type silica, Tocopheryl acetate, Sodium fluoride, } \\
\text { Sodium monofluorophosphate, Sodium Pyrophosphate }\end{array}$ & Korea \\
\hline \multirow[t]{3}{*}{$\begin{array}{l}\text { Toothpaste for } \\
\text { pregnant women }\end{array}$} & $\begin{array}{l}\text { Experimental } \\
\text { group }\end{array}$ & Mommiracle toothpaste & $\begin{array}{l}\text { Precipitated calcium carbonate, Calcium hydrogen } \\
\text { phosphate, Aminocaproic acid, Allantoine } \\
\text { chlorohydroxy aluminum }\end{array}$ & Korea \\
\hline & & $\begin{array}{l}\text { Dr. Jeninmothers } \\
\text { toothpaste }\end{array}$ & $\begin{array}{l}\text { Silicon dioxide, Aminocaproic acid, Allantoine } \\
\text { chlorohydroxy aluminum }\end{array}$ & Korea \\
\hline & & Tntnmoms toothpaste & Silicon dioxide, Tocopheryl acetate & Korea \\
\hline
\end{tabular}


cavity care [LG living health, Seoul, Korea], Cliden original [LG living health]), and three types of pregnant women's dentifrices were selected as experimental groups, including (Mommiracle toothpaste [Mommiracle, Anseong, Korea], Dr. Jeninmothers toothpaste [Zeniton, Seoul, Korea], and Tntnmoms toothpaste [Tntnmoms, Seongnam, Korea]) (Table 1).

\section{Research methods}

\section{1) Sample production}

After removing the attachment and coloring of 60 selected bovine teeth using an ultrasonic scaler, the samples were produced by cutting and polishing using a diamond disk of $10.5 \times 10 \mathrm{~mm}$ dimensions. Ten samples were allocated to each of the three types of general and pregnant women's dentifrices.

\section{2) Artificial biofilm formation and removal}

On the labial surface of bovine teeth, dental plaque in the actual oral environment applied a carbohydrate porridge for 24 hours to reproduce the calming form and then formed a rough surface. Afterwards, a cotton ball with disclosing agent evenly buried on the labial surface of the sample was applied until $100 \%$ of the area of the bovine teeth was applied using pincette to make it easier to understand the status of the removal of the acidic differential plaque. Next, the same operator used an

Table 2. Residual Amount of Plaque between Bovine Tooth in General Dentifrices

\begin{tabular}{crrr}
\hline \multirow{2}{*}{ No. } & \multicolumn{3}{c}{ Residual area of disclosing agent $(\%)$} \\
\cline { 2 - 4 } & Perio & Cliden & 2080 \\
\hline 1 & 7.23 & 25.05 & 18.20 \\
2 & 22.32 & 0.13 & 6.31 \\
3 & 12.40 & 1.04 & 17.74 \\
4 & 32.80 & 4.68 & 6.06 \\
5 & 4.35 & 7.54 & 14.83 \\
6 & 0.46 & 31.56 & 1.84 \\
7 & 4.24 & 11.81 & 3.10 \\
8 & 5.98 & 2.09 & 7.13 \\
9 & 5.61 & 4.41 & 3.18 \\
10 & 21.66 & 6.20 & 6.29 \\
Average & 11.71 & 9.45 & 8.47 \\
& & 9.88 & \\
\hline
\end{tabular}

electric toothbrush to brush for 30 seconds per sample, then the finished sample was placed in a beaker containing tap water and shaken in a circle to remove dentifrices and other residues from leaving 15 times of water rinsing. Afterwards, the sample was dried and used in the experiment.

\section{3) Calculation of artificial biofilm residual amount}

After fixing the DSLR camera at approximately $10 \mathrm{~cm}$ distance perpendicular to the sample, only the sample was replaced to maintain the angle and distance. For measure the residual amount of the disclosing agent, the area of the disclosing agent relative to the total area of the sample was analyzed and quantified using the ImageJ program (ImageJ bundled with 64-bit Java 1.8.0_172; National Institute of Mental Health, Bethesda, MD, USA).

\section{4) Statistical analysis}

Statistical analysis was performed using the IBM SPSS Statistics 26.0 program (IBM Corp., Armonk, NY, USA). For analyzing the significant difference in the residuals between the general and pregnant women's dentifrices, the Kruskal-Walis test was performed, and the Mann-Whitney test was conducted to analyze the significant difference in the residuals between the general and pregnant women's dentifrices. All tests were conducted below a significant level of 0.05 .

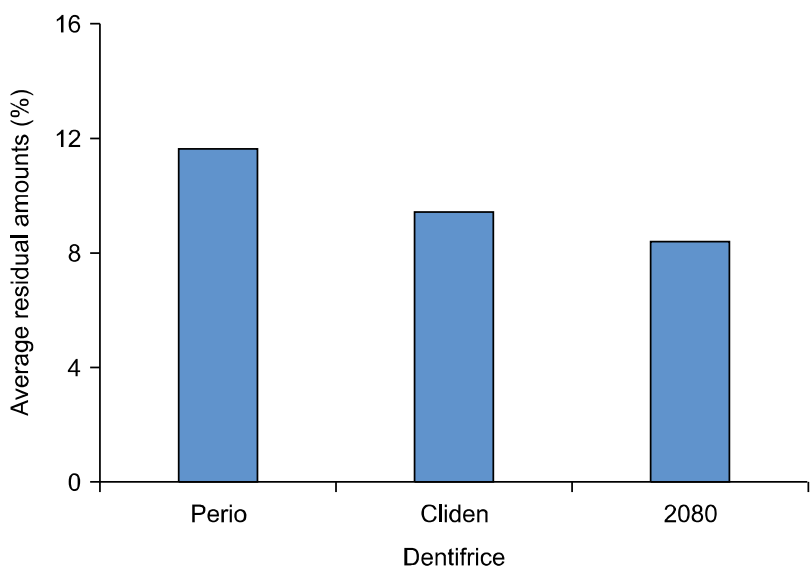

Fig. 1. General dentifrice's residual amounts comparison. The average residual amounts of dental plaque was $11.71 \%$ for Perio, 9. $45 \%$ for Cliden, and $8.47 \%$ for 2080 . 
Table 3. Comparison of Dentifrice's Residual Amount between General Dentifrice and Pregnant Women's Dentifrice

\begin{tabular}{lccccc}
\hline \multicolumn{1}{c}{ Classification } & $\mathrm{n}$ & Mean \pm standard deviation & Minimum value & Maximum value & $\mathrm{p}$-value \\
\hline General dentifrice & 30 & $9.88 \pm 9.08$ & 0.13 & 32.80 & 0.817 \\
Pregnant women's dentifrice & 30 & $13.04 \pm 11.46$ & 0.74 & 43.38 & 0.903 \\
\hline
\end{tabular}

p-value of general dentifrice-pregnant women's dentifrice $=0.260$.

The data were analysed by Kruskal-wallis.

Table 4. Residual Amount of Plaque between Bovine Tooth in Pregnant Women's Dentifrice

\begin{tabular}{cccc}
\hline \multirow{2}{*}{ No. } & \multicolumn{3}{c}{ Residual area of disclosing agent $(\%)$} \\
\cline { 2 - 4 } 1 & Jeninmothers & Mommiracle & Tntnmoms \\
2 & 14.57 & 33.46 & 4.89 \\
3 & 5.37 & 0.74 & 5.36 \\
4 & 6.72 & 8.04 & 11.93 \\
5 & 5.08 & 3.20 & 13.65 \\
6 & 7.80 & 5.59 & 5.57 \\
7 & 5.74 & 19.75 & 34.78 \\
8 & 26.04 & 23.31 & 5.17 \\
9 & 43.38 & 4.83 & 5.84 \\
10 & 1.09 & 11.33 & 4.50 \\
Average & 23.66 & 16.09 & 33.59 \\
& 13.95 & 12.63 & 12.53 \\
\hline
\end{tabular}

\section{Results}

1. Comparative analysis on the residuality of the dental plaque of the three types of general dentifrices

The analysis was performed using the ImageJ program to compare the dental plaque residue of the three types of general dentifrices. The results demonstrated that the average residual dental plaque was $11.71 \%$ for Perio, 9.45\% for Cliden, and $8.47 \%$ for 2080 with an average residual plaque of approximately $9.88 \%$ for the three types of general dentifrices (Table 2, Fig. 1). The KruskalWallis test for comparing the residual dental plaque of general dentifrices revealed that the significance probability was 0.817 and residual dental plaque of the three types of general dentifrices was not significantly different (Table 3 , $\mathrm{p}>0.05)$.

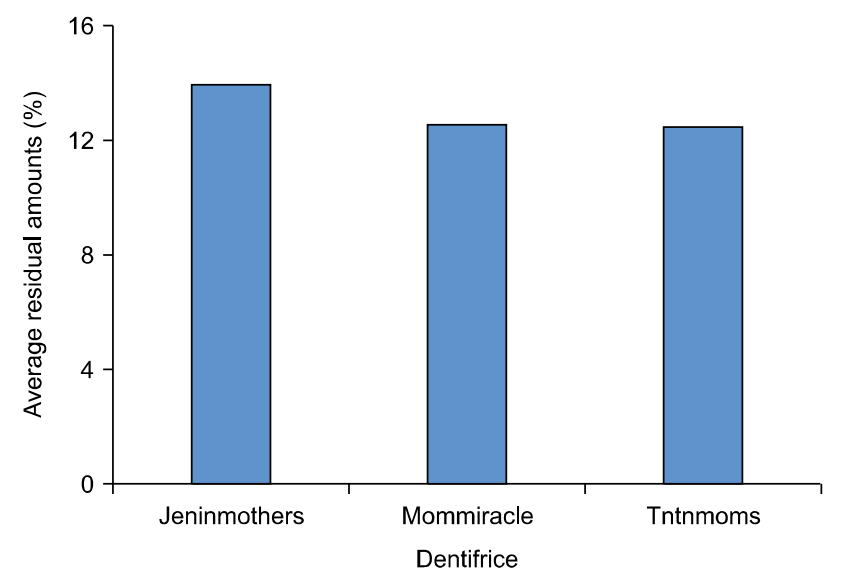

Fig. 2. Pregnant women's dentifrice's residual amounts comparison. The average residual amounts of dental plaque was $13.95 \%$ for Jeninmothers, $12.63 \%$ for Mommiracle, and $12.53 \%$ for Tntnmoms.

\section{Comparative analysis on the residuality of the dental plaque of the three types of pregnant women's dentifrices}

For comparing the residual dental plaque of the three types of pregnant women's dentifrices, the ImageJ program was used. As a result, the average residual dental plaque was $13.95 \%$ for Jeninmothers, $12.53 \%$ for Tntnmoms, and $12.63 \%$ for Mommiracle, with an average residual plaque of approximately $13.04 \%$ for the three types of pregnant women's dentifrices (Table 4, Fig. 2). The Kruskal-Wallis test for comparing the residual dental plaque of pregnant women's dentifrices revealed that the significance probability was 0.903 and residual dental plaque of the three types of pregnant women's dentifrices was not significantly different (Table $3, \mathrm{p}>0.05$ ).

\section{Comparative analysis on the residuality of the dental plaque of the general and pregnant women's dentifrices}

ImageJ program was used to compare and analyze the residual amount of dental plaque in general and pregnant 


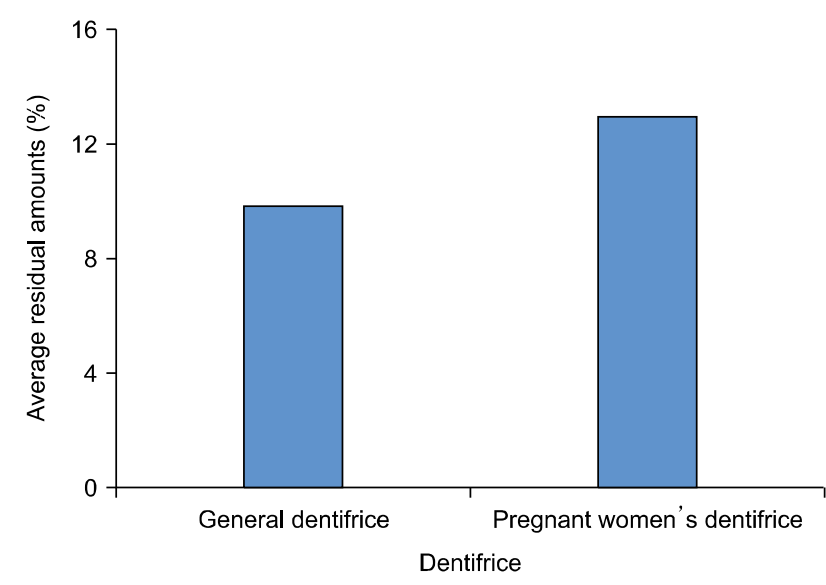

Fig. 3. Comparison of general and pregnant women's dentifrice's residual amounts. The average residual amount of dental plaque was $9.88 \%$ for general dentifrice and $13.04 \%$ for pregnant women's dentifrice. The difference between the two groups was 3. $16 \%$.

women's dentifrices. Comparing the two groups, the average residual of the general dentifrices was $9.88 \%$ and that of the pregnant women's dentifrices was $13.04 \%$, indicating that the residual amount for pregnant women's dentifrices were $3.16 \%$ higher than the general dentifrices (Fig. 3). An analysis of the mean residual values of general and pregnant women's dentifrices using a Mann-Whitney test revealed that the mean residuals of general and pregnant women's dentifrices were not statistically significant (Table 4, $\mathrm{p}>0.05$ ).

\section{Discussion}

For pregnant women requiring special oral health care, tooth brushing, a self-oral health care method, is critical, and dentifrices used to efficiently clean the teeth surfaces are essential elements in tooth brushing ${ }^{11)}$. The main ingredients of dentifrices are polishing agents, detergents, binders, wetting agents, fluoride, and other ingredients are added to prevent dental caries. Among these components, SLS is a synthetic detergent that is highly accessible and economical with the components of other dentifrices ${ }^{4)}$; however, as reported in Elder's research ${ }^{8)}$, it can be easily absorbed through the skin and causes chronic diseases if accumulated in the body for a long period. Malin and Till $^{10)}$ also reported that exposure to fluoride in the formation of the fetus could lead to cognitive impairment.
The pregnant women's dentifrices currently available in the market do not include chemicals that can damage the human body, such as SLS and fluoride and are being replaced by other natural ingredients. However, the preceding papers are very insufficient to study pregnant women's dentifrices, and we think it is also necessary to verify that pregnant women's dentifrices, which do not include these chemicals, perform the auxiliary functions of the toothbrush correctly. Thus, in this study, the dental plaque removal effects of general and pregnant women's dentifrices were compared.

$\mathrm{Choi}^{12)}$ noted that there was no significant difference in the dental plaque removal capability between each group in the three types of general dentifrices and that all three species demonstrated a slight decrease in the periodontal pocket depth after 4 weeks; however, the difference was not statistically significant. The study by Kim et al. ${ }^{13)}$ also reported that the surface hardness between NaF-containing dentifrices did not show significant difference and there was no significant difference in remineralisation of enamel. In this study, carbonated porridge was applied to the labial surface of Bovine teeth to form acidic dental plaque and disclosing agent was applied to find the effect of removing the dental plaque of the three types of general dentifrices. Afterward, it was processed with three types of general dentifrices and photographed using a DSLR camera, and the residual amount of dental plaque was compared and analyzed using ImageJ program. As a result, the average residual dental plaque was $11.71 \%$ for Perio, $9.45 \%$ for Cliden and $8.47 \%$ for 2080 . The average value between general dentifrices was slightly different, but it was found that there was no statistically significant difference with a significant probability of 0.817 . The results of this study were similar to those of $\mathrm{Choi}^{12)}$ and Kim et al. ${ }^{13)}$.

Meanwhile, in the study by Shim et al. ${ }^{14)}$, the change in the number of bacteria in the mouth according to the presence or absence of SLS in the dentifrices showed no significant difference. On comparing the dental plaque removal effects of D. I. Y. dentifrices that do not contain SLS with general dentifrices, the results demonstrated that approximately $14 \%$ of the dental plaque removal effects were better than those of D. I. Y. dentifrices but were not 
statistically significant ${ }^{15)}$. Jeong et al. ${ }^{16)}$ observed that lower the SLS content in the dentifrices, the higher was the secretion of the saliva and the higher the SLS content in the dentifrices, the lower the saliva content. Jeong ${ }^{17)}$ also concluded that the higher the content of SLS, the lower the saliva secretion, causing oral dryness and bad breath. The study by Jensen and Barkvoll ${ }^{18)}$ also emphasized that patients with low saliva secretion should be prescribed dentifrices that do not contain SLS. In a survey conducted by Lee and $\mathrm{Choi}^{19)}$ in 180 pregnant women on oral health, $59.4 \%$ reported of 'sometimes feel bad' and $56.7 \%$ of 'sometimes feel oral dryness'. In this study, experiments were conducted in the same method as general dentifrices to identify the elimination effect of dental plaque in three types of pregnant women's dentifrices. The average residual dental plaque residuals was $13.95 \%$ for Jeninmothers, $12.53 \%$ for Tntnmoms, and $12.63 \%$ for Mommiracle. There were slight differences in the mean values between the general and pregnant women's dentifrices, but the significance probability was 0.903 , indicating that there were no statistically significant differences. On comparing the effect of elimination of dental plaque between general and pregnant women's dentifrices, the average residual plaque of the three types of general dentifrices was $9.88 \%$, and that of the three types of pregnant women's dentifrices was $13.04 \%$. However, the significant probability of general and pregnant women's dentifrices was 0.260 , indicating no statistically significant difference. These results proved that pregnant women's dentifrices has a dental plaque removal effect similar to that of general dentifrice even though it does not contain SLS with excellent cleaning power and fluoride for preventing dental caries unlike general dentifrice. Additionally, grapefruit seed extract, a component contained in the two pregnant women's dentifrices was used in this study. Lee et al. ${ }^{20)}$ used only grapefruit seed extract to identify antibacterial effects on the causative bacteria of cavities, gingivitis, periodontitis, and candidiasis, and reported the physical and chemical properties to be relatively stable when mixed in the dentifrices prototype. Therefore, the results of this study suggest that pregnant women should use pregnant women's dentifrices that do not contain SLS. However, in this study, a dental plaque was formed with a carbohydrate porridge to reproduce the oral environment, but it did not apply to the oral cavity, so it would be desirable to experiment with pregnant women in the next study.

Correct brushing with dentifrices is critical for pregnant women, along with the general public, elderly, and disabled, and maintenance of healthy periodontal tissue during pregnancy is highly desirable in terms of time spent with the child and economics after childbirth ${ }^{21)}$. Therefore pregnant women who use pregnant women's dentifrices will be able to prevent or inhibit progress of plaque during pregnancy if they continue to use the same dentifrices along with the correct brushing. Since there is no significant difference in general and pregnant women's dentifrices, it is recommended for pregnant women with severe emesis gravidarum when brushing teeth to use pregnant women's dentifrices.

\section{Notes}

\section{Conflict of interest}

No potential conflict of interest relevant to this article was reported.

\section{Ethical approval}

This article does not require an IRB because it used tissue that was thrown away from the carcasses of animals.

\section{Author contributions}

Conceptualization: Do-Seon Lim. Data acquisition: Ji-Eun Lee. Formal analysis: Ye-Eun Kim, Seong-Eun Ko, Da-Eun Sa. Funding: Do-Seon Lim. Supervision: Do-Seon Lim. Writing-original draft: Se-Yoon Jeon. Writingreview \& editing: Do-Seon Lim.

\section{ORCID}

Ye-Eun Kim, https://orcid.org/0000-0002-1534-1567

Seong-Eun Ko, https://orcid.org/0000-0003-2806-1510

Da-Eun Sa, https://orcid.org/0000-0001-9691-113X

Ji-Eun Lee, https://orcid.org/0000-0002-5609-6190

Se-Yoon Jeon, https://orcid.org/0000-0002-7447-7843

Do-Seon Lim, https://orcid.org/0000-0003-4602-3323 


\section{References}

1. Lee SJ, Choi GY: A study on the oral health behavior status and oral health awareness of pregnant women -demographic socialogical variables-. J Korea Acad-Ind Cooper Soc 12: 5049-5055, 2011. https://doi.org/10.5762/KAIS.2011.12.11.5049

2. Jang KA, Kim KO, Lee SO: Comparing oral health care awareness and practice in pregnant women with and without oral health education experience. J Korean Soc Matern Child Health 20: 169-177, 2016. https://doi.org/10.21896/jksmch.2016.20.2.169

3. Park JH, Lee KS: Comparison of oral care patterns before and during the pregnancy. J Dent Hyg Sci 11: 273-278, 2011.

4. Kang BW, Kim KS, Park MS, et al.: Preventive dentistry. Komoonsa, Seoul, pp.102-108, 2017.

5. Shin KY, Park CW, Lee CH: Perturbation and recovery of the skin barrier function after tape stripping and sodium lauryl sulfate irritation. Korean J Dematol 38: 183-190, 2000.

6. Fakhry-Smith S, Din C, Nathoo SA, Gaffar A: Clearance of sodium lauryl sulphate from the oral cavity. J Clin Periodontol 24: 313-317, 1997. https://doi.org/10.1111/j.1600-051X.1997.tb00763.x

7. Herlofson BB, Barkvoll P: Sodium lauryl sulfate and recurrent aphthous ulcers. A preliminary study. Acta Odontol Scand 52: 257-259, 1994. https://doi.org/10.3109/00016359409029036

8. Elder RL: Final report on the safety assessment of sodium lauryl sulfate and ammonium lauryl sulfate. J Am Coll Toxicol 2: 127-181, 1983. https://doi.org/10.3109/10915818309142005

9. Bashash M, Marchand $\mathrm{M}, \mathrm{Hu} \mathrm{H}$, et al.: Prenatal fluoride exposure and attention deficit hyperactivity disorder (ADHD) symptoms in children at 6-12 years of age in Mexico City. Environ Int 121(Pt 1):658-666, 2018. https://doi.org/10.1016/j.envint.2018.09.017

10. Malin AJ, Till C: Exposure to fluoridated water and attention deficit hyperactivity disorder prevalence among children and adolescents in the United States: an ecological association. Environ Health 14: 17, 2015. https://doi.org/10.1186/s12940-015-0003-1

11. Lee KY, Won BY: A study on the oral health care of pregnant women in a region. J Korean Acad Dent Hyg Educ 9: 1-14,
2009.

12. Choi SM: The effect of several toothpastes on the sulcus bleeding index, plaque index and gingival keratinization. $\mathbf{J}$ Korean Dent Assoc 22: 417-422, 1984.

13. Kim JH, Jeong SS, Choi $\mathrm{CH}$, Hong SJ: Fluoride concentration of commercial dentifrices and effect of fluoride containing dentifrices on artificial enamel caries. J Korean Acad Dent Health 30: 56-66, 2006.

14. Shim YS, Jeong MA, Jeong SH: The change of salivary and oral bacteria amount by composition of SLS contents of toothpaste. J Korea Acad-Ind Cooper Soc 11: 3341-3346, 2010. https://doi.org/10.5762/KAIS.2010.11.9.3341

15. Jung YS, Lim SH, Oh SH, Kang KH, Koong HS, Hwang SJ: Effect of dental plaque removal and preference about D.I.Y dentifrices. J Korean Acad Dent Hyg Educ 10: 311-322, 2010.

16. Jeong HY, Kim YS, Jeong MA: Variations of oral cavity environment according to sodium lauryl sulfate concentration of toothpaste. J Korea Contents Assoc 10: 240-248, 2010. https://doi.org/10.5392/JKCA.2010.10.8.240

17. Jeong MA: Relationship of sodium lauryl sulfate content to the effects of dentifrice on halitosis. J Korea Acad-Ind Cooper Soc, 902-905. Retrived November 9, 2020, from http://www.dbpia.co.kr/journal/articleDetail?nodeId=NODE 07216856(2010).

18. Jensen JL, Barkvoll P: Clinical implications of the dry mouth: oral mucosal diseases. Ann N Y Acad Sci 842: 156-162, 1998.

https://doi.org/10.1111/j.1749-6632.1998.tb09643.x

19. Lee SJ, Choi GY: A study on the oral health status and oral health awareness of pregnant women. J Korea Acad-Ind Cooper Soc, 128-130. Retrived November 9, 2020, from http://www.dbpia.co.kr/journal/articleDetail?nodeId=NODE 07216424(2009).

20. Lee BB, Ha YM, Shin SH, et al.: Antimicrobial activity of test dentifrice product containing grapefruit seed extract and processed sulfur solution against oral pathogens. J Life Sci 19: 956-962, 2009. https://doi.org/10.5352/JLS.2009.19.7.956

21. Heo AR, Song KS, Cha EJ, Kim KA, Shon HS: Relationship of oral health status and oral health behaviors to the Oral Health Impact Profile (OHIP) of the pregnant women. J Korea Contents Assoc 16: 81-89, 2016. https://doi.org/10.5392/JKCA.2016.16.08.081 